\title{
DIEZ MODELOS RELACIONADOS CON LA PRÁCTICA REFLEXIVA
}

\author{
TEN MODELS RELATED TO \\ REFLECTIVE PRACTICE
}

Ingrid Eugenia Cerecero Medina

Doctora en Ciencias Sociales. Profesora de Instituciones Políticas y Manejo del Poder en el Doctorado de Imagen Pública, Colegio de Imagen Pública. Representante de la Plataforma Internacional de Práctica Reflexiva en México. Investigadora en el área de Educación y Cultura.

iecerecerom@uaemex.mx; ingridecm@yahoo.com

\section{RESUMEN}

El presente artículo constituye una investigación documental que describe algunos antecedentes de la práctica reflexiva, fundamentalmente acerca de la reflexión. Posteriormente, se vincula la práctica reflexiva con la investigación-acción y se presentan diez modelos que ejemplifican diversas opciones, que aun cuando provienen de distintas teorías o métodos, pretenden ser alternativas para ejercer una praxis que permita la mejora tanto de la práctica profesional, como de quien la lleva a cabo. 
Se estima que la práctica reflexiva puede alentar el aprendizaje y la transformación no solo del ejercicio profesional, sino también del sujeto que la ejerce, pudiendo emplear el modelo que convenga a sus intereses y a la profundidad de cambio que quiera experimentar. Los profesionales necesitan ser reflexivos, conocerse a ellos mismos, conocer su trabajo, su contexto y provocar cambios para el bien común.

Palabras clave: práctica profesional, pensamiento crítico, investigación-acción.

\section{ABSTRACT}

The article provides a documentary research that supports the reflective practice approach, closely linked to action research. It also reviews ten models that represent alternatives for the improvement of professional practice even when these models differ in their theoretical framework or methodological strategies, they have as a common ground the reflection on daily practices. The article stresses the fact that reflective practice not only promotes advancement at the professional level, but transforms at the personal level, the professional practicing it.

Keywords: Professional practice, Critical thinking, Action research.

\section{INTRODUCCIÓN}

En el ámbito educativo, el pensamiento crítico y la reflexión constituyen dos de los retos de este siglo. Sin embargo, no puede exigirse un pensamiento crítico ni reflexivo en los estudiantes, si los profesores o profesionales de cualquier área, no lo tienen. Es 
por ello, que la práctica reflexiva del docente es un punto en el que la educación puede enfocarse para mejorar los procesos de enseñanza-aprendizaje.

Aun cuando este artículo va dirigido principalmente a docentes, se decidió generalizar la información, considerando que cualquier profesional puede ser beneficiado de llevar una práctica reflexiva.

El objetivo de esta investigación documental es presentar de forma resumida algunos modelos relacionados con la práctica reflexiva que permitan al profesional educativo o de cualquier otra área, darse cuenta que, a través de la reflexión, puede lograrse el entendimiento de una situación o un problema; mientras que, por medio de la práctica reflexiva, puede mejorarse - e incluso transformarse- el quehacer de quien la lleva a cabo.

Por lo tanto, en un primer momento, se inicia con un breve recuento de algunas ideas expresadas por diversos autores o personajes reconocidos, acerca de la reflexión, reuniendo y relacionando palabras clave que constituyen elementos, características o estrategias para reflexionar.

Con base en la investigación documental, se conceptualiza la reflexión y la práctica reflexiva, así como características de las mismas, fusionando la información citada por diversos autores.

Se incluye una descripción breve y relación de algunos modelos con la práctica reflexiva - sin que necesariamente todos estén clasificados dentro de este rubro-, tomando uno o más de los modelos de la investigación-acción, del aprendizaje experiencial, de la teoría de la actividad, o bien de la práctica reflexiva.

Finalmente, se expresan algunas conclusiones después de revisar los modelos descritos, considerando que cada uno puede ser útil al lector, independientemente de su profesión y dependiendo del objetivo y profundidad con que se interese en llevar a cabo el proceso reflexivo.

El propósito fundamental del texto es crear consciencia de que existen diversas opciones para llevar a cabo una práctica reflexiva, que el 
lector conozca o revise algunas de estas opciones, las compare, y de ser posible, las pruebe y observe con cuál se identifica para, idealmente, decidir emplear alguna que se adecue - continua y sistemáticamente- a sus necesidades.

\section{ANTECEDENTES}

Antes de estudiar los modelos de práctica reflexiva o aquéllos relacionados con esta, mencionaré que, a lo largo del tiempo, diversos autores han tratado la relevancia de la reflexión en distintos ámbitos. Aun cuando se reconoce a Donald Schön (1998) como el primero en acuñar el término de "práctica reflexiva» como se conoce actualmente, algunos filósofos a través de la historia han señalado la importancia de la reflexión o de alguno de sus elementos, por ejemplo:

Lao Tsé (Atkinson, 2011) mencionó, en sus escritos, que para lograr el Tao o camino, era necesaria la reflexión, además de la meditación, la paz y la armonía.

Sócrates (Atkinson, 2011) estaba a favor de debatir, discutir y cuestionar las creencias de la gente con la finalidad de entender la realidad. Estos pasos pueden ser considerados parte de un proceso reflexivo. Además, con su propuesta — conocida como «método socrático»—, empleando la ironía, se puede hacer ver, al que cree que sabe, que está equivocado; o por medio de la «mayéutica», podemos lograr que quien sabe y que cree que no sabe, se dé cuenta de su conocimiento, y finalmente, mediante la «aletheia» se puede sacar a la luz lo que es verdad.

Platón (Atkinson, 2011) al considerar que el mundo real es el de las ideas y que el conocimiento verdadero proviene de la razón, permite darnos cuenta que la reflexión existe a partir de un pensamiento razonado y objetivo, intentando dejar fuera los sentidos y las sensaciones.

Aristóteles (Atkinson, 2011) destaca la observación como un proceso a través del cual se puede descubrir la realidad. A diferencia de Platón, considera que no es solo la razón sino también son los sentidos, los que nos permiten conocer al mundo. De esta manera, se rescata que 
la observación es un elemento necesario para reflexionar y que los sentidos pueden apoyar nuestras teorías.

John Locke (Atkinson, 2011) toma como base de la reflexión a la experiencia debido a que considera que todos somos distintos y, por lo tanto, lo que vivimos y percibimos, así como los contextos en que nos desenvolvemos y las situaciones que enfrentamos, también lo son. Por lo tanto, podemos considerar a la experiencia como otro elemento más del proceso reflexivo.

Gottfried Leibniz (Atkinson, 2011) da mayor importancia a la razón (verdad de razón) que a la experiencia (verdad de hecho), aun cuando considera a ambas necesarias para adquirir conocimiento. Aquí destacamos el análisis de la razón y de las experiencias para reflexionar.

René Descartes propuso el método cartesiano de la duda para llegar a la verdad y a través de este, de acuerdo con el Diccionario de Psicología Científica y Filosófica (2015), Descartes propone dudar de todo lo que se cree o dice, sin importar quién lo haya expuesto ni su autoridad o vigencia; incluso se debe dudar de los sentidos y de todos los cuerpos físicos, estimando que si la mente es inmaterial, autoconsciente y una sustancia pensante, puede crear todo lo demás; de ahí su frase: «Pienso, luego existo», es decir, no puede afirmarse la veracidad de algo, a menos que se tenga absoluta certeza a través de evidencias; para intuir la evidencia de una afirmación, esta debe descomponerse en afirmaciones simples (análisis) y, después, unirse por medio de deducciones para ver su relación lógica (síntesis), hasta finalmente verificar que no haya error en los pasos anteriores.

Como puede observarse, Descartes toma en cuenta la intuición y la deducción, estableciendo la necesidad de superar varios obstáculos: el engaño que pueden producir los sentidos (no saber si estamos soñando o despiertos); las creencias religiosas (nos hacen creer lo que no es); nuestros propios pensamientos incorrectos. Con base en lo anterior, entendemos que el proceso de reflexión inicia con la acción de dudar y requiere cuestionar, analizar, sintetizar y evaluar.

Immanuel Kant (Atkinson, 2011) señala que para entender cómo funcionan las cosas en el mundo se requiere sensibilidad o intuición, 
entendida como la capacidad de estar en contacto con las cosas en el aquí y el ahora; ello supone además el entendimiento, el cual identifica como la capacidad de saber los conceptos de las cosas y cuándo utilizarlos. Nuevamente se encuentra que la experiencia y la razón son elementos necesarios para la reflexión.

Kant establece que la reflexión es el estado del espíritu (Lisón, 1991) por el que nos encontramos en la disposición de descubrir las condiciones de carácter subjetivo para formar un concepto. Como Muñoz y Velarde (2000) lo señalan, Kant refiere al modo en que podemos conocer los objetos a través de la experiencia que los sujetos tienen con cada objeto de manera que, a través de la subjetividad, se busque la objetividad por medio de la interpretación que se hace del objeto mismo.

Edmund Husserl (García, 1999) de acuerdo con su propuesta de fenomenología trascendental, explica que el sujeto interpreta el mundo para darle sentido. Esto es, vemos la realidad de acuerdo con nuestras percepciones que son distintas para cada persona según sus experiencias, sensaciones, recuerdos, visualización e imaginación, pudiendo actualizarlas conforme a la atención o reflexión que el sujeto tenga del objeto.

De acuerdo con Husserl deben dejarse a un lado todos los presupuestos; esto es, entrar en un estado mental que evite juzgar o tomar posición, para acabar con la enajenación del sujeto, lo que este autor llama epojé.

Por lo anterior, otro elemento de la reflexión es el darse cuenta que, lo que para un individuo es cierto, no forzosamente lo es para los demás; sino que son solo sus percepciones. Por lo que en lugar de juzgar los hechos, el sujeto debe partir de su observación, sin etiquetar lo sucedido, liberándose de prejuicios y permitiendo proporcionar un nuevo sentido y significado a los objetos y hechos experimentados.

Martin Heidegger (León, 2012) pondera la interpretación basándola en la comprensión del objeto. A partir del pensamiento de este autor, consideramos que cuando se reflexiona se necesitan de estos elementos para aplicarlos no solo al objeto, sino también a los sujetos que intervienen. 
John Dewey (1993) expone que el pensamiento reflexivo siempre tiene un propósito y una conclusión, por lo que implica una secuencia de ideas y sus consecuencias. Asimismo, señala que este proceso requiere de curiosidad, sugerencias ${ }^{1}$, lógica, flexibilidad, variedad, orden, control, una mente abierta, entusiasmo y aceptación de la responsabilidad que conlleva.

Además, Dewey propone seis fases para el pensamiento reflexivo, las cuales no necesariamente siguen un orden establecido, ni tienen que llevarse a cabo todas, a menos que se considere necesario. Estas fases son: sugerencia, intelectualización, hipótesis, razonamiento, comprobación de la hipótesis a través de la acción u observación directa, y visión del futuro. Asimismo, destaca la referencia al pasado, que considera ya implícita en las sugerencias, pues estas dependen de lo ya experimentado. Además, considera factores subordinados como son los juicios (que incluyen las funciones de análisis y síntesis para poder emitirlo), la comprensión (que deriva de la investigación o de la práctica) y el concepto (como modelos de referencia estandarizados).

Además de lo anterior, Dewey propone un método sistemático para la reflexión que evita generalizaciones y abarca la observación guiada por una hipótesis, el análisis, la recopilación y comparación de datos, su comparación y contraste; y la construcción deliberada de datos, a partir de una variación experimental de las condiciones.

Desde el punto de vista de este filósofo, la reflexión permite el cuestionamiento de las experiencias vividas, por lo que es un proceso de indagación, observación e investigación que debe ser continuo, flexible, abierto y que evita las ideas preconcebidas. Podría afirmarse que Dewey, con esta concepción que hace de la reflexión, ya está refiriendo una práctica reflexiva al indicar que la reflexión debe ser un método sistemático o un proceso continuo.

1 Dewey define las sugerencias como ideas que pueden ser controladas, tienen un significado y son fuentes de pensamiento. 
Maurice Merleau Ponty (Atkinson, 2011) enfatiza que debemos reaprender a observar nuestras experiencias, las cuales pueden estar llenas de contradicciones; asimismo, deben evitarse los presupuestos que impiden ver la realidad.

De acuerdo con Anthony Giddens (Torres y Miranda, 2013), los individuos somos seres activos y participativos que transformamos nuestras acciones. La reflexión es necesaria para que la acción posea significado, y pueda modificarse o repetirse de forma consciente.

Muñoz y Velarde (2000) entienden a la reflexión como un constante retorno sobre símismo y la clasifican como un método de la teoría del conocimiento que requiere de observación, de la búsqueda de la relación sujeto-objeto, del análisis y la síntesis.

Torres y Miranda (2013), Dewey (1989), Schütz (1974), Flavell y Wellman (1977) consideran a la reflexión como un proceso del pensamiento que parte de la metacognición y que tiene como propósito producir, reproducir o transformar acciones o comportamientos.

Reuniendo las ideas expresadas por los autores antes mencionados, se extraen algunas palabras clave relacionadas con la reflexión o algunas como posibles elementos de la misma o estrategias para llevarla a cabo, entre ellas están:

- Debate o discusión.

- Cuestionamiento.

- Ironía, mayéutica y / o aletheia.

- Razonamiento objetivo.

- Observación.

- Duda.

- Análisis de la razón y de la experiencia.

- Síntesis.
- Intuición.

- Deducción.

- Entendimiento.

- Interpretación.

- Reaprendizaje.

- Epojé.

- Comprensión.

- Significado.

- Transformación. 
- Relación sujeto-objeto.

- Pensamiento.

- Proceso.

- Propósito.

- Curiosidad.

- Entusiasmo.

- Sugerencias.

- Consecuencias.

- Juicios.

- Conceptos.
- Visión del futuro.

- Referencia al pasado.

- Lógica.

- Flexibilidad.

- Variedad.

- Orden.

- Control.

- Apertura.

- Responsabilidad.

- Continuidad.

A partir de lo expuesto anteriormente, se entiende que la reflexión es un proceso complejo que requiere de cuestionar y cuestionarse para buscar el significado de un acontecimiento, analizar y entender al propio sujeto, a otro(s) sujeto(s), a un objeto o una situación; dejando a un lado los prejuicios, para emitir un juicio lo mayormente objetivo y que permita al sujeto observar, analizar y sintetizar las experiencias y los conocimientos pasados y presentes para comprender, resolver, resignificar, transformar o proyectar sus resultados en un acontecimiento similar futuro.

Una vez habiendo establecido algunas conceptos relacionados con la reflexión por diversos autores a lo largo del tiempo, y llegando a nuestra propia idea de lo que es la reflexión, se encuentra otro antecedente fundamental de la práctica reflexiva, que es la investigación-acción.

Se reconoce a Kurt Lewin (1946) como el primero en proponer la investigación-acción, entendiéndola como una propuesta metodológica para resolver problemas significativos en diversas áreas, a través de 
un cuestionamiento reflexivo, donde se involucra a los participantes como agentes de cambio, con el propósito de mejorar una situación en su propia práctica o en su contexto social. Existen diversos modelos de investigación-acción muy similares a los de la práctica reflexiva, por lo que consideramos a los primeros como la base de los segundos.

Tanto la investigación-acción, como la práctica reflexiva, recurren a la reflexión de la experiencia para transformar una situación. Sin embargo, la práctica reflexiva centra su atención en el profesional que la lleva a cabo. Es decir, se aplica específicamente respecto del profesional mismo y a su desarrollo, o bien, a las actividades que realiza o intervienen directamente en su trabajo; mientras que la investigación-acción propone la solución de uno o más problemas identificados, utilizando una metodología rigurosa de investigación estratégica y en la mayoría de los casos proponiendo cambios sociales e involucrando a distintos actores, no solamente a quienes lo proponen.

Por otro lado, como ya se mencionó, se atribuye a Donald Schön (1992) el término de práctica reflexiva, así como el de formación del profesional reflexivo, destacando la existencia de la reflexión en la acción (durante la misma) y la reflexión sobre la acción (posterior a lo que se hizo). Se considera que una reflexión en la acción es una actividad pre-reflexiva, es decir, crea consciencia del suceso sin profundizar en este, es inintencional y superficial; mientras que la reflexión sobre la acción es profunda e intencional.

La práctica reflexiva, dentro del ámbito educativo ha sido definida por Domingo (2012) como:

[...] una metodología de formación en que los elementos principales de partida son las experiencias de cada docente en su contexto y la reflexión sobre su práctica. Se trata de una opción formativa que parte de la persona y no del saber teórico, que tiene en cuenta la experiencia personal y profesional para la actualización y la mejora de la tarea docente.

En esta definición se encuentra como elemento principal la reflexión de la experiencia por encima de la teoría con una intencionalidad de actualización profesional. 
Barnett (1992), al igual que Domingo (2012), explican a la práctica reflexiva como un procedimiento de formación y, junto con Moon (1999), apoyan la postura de Schön destacando el proceso reflexivo durante o sobre las acciones o experiencias en un contexto dado; mientras que Perrenoud (2007), Lantolf (2007), Pedroza (2014) y Cerecero (2018) consideran que, para que exista una práctica reflexiva, debe haber forzosamente una reflexión sobre la acción en la que se revisen los sucesos y estos se soporten con las teorías para llevar a cabo un análisis más profundo.

Con base en lo anterior, la práctica reflexiva puede conceptualizarse como un proceso cíclico y sistemático de análisis y deliberación, respecto del ejercicio profesional, que posibilita una toma de decisiones fundamentada y que contribuye al desarrollo o transformación del profesional.

Reuniendo algunos de los planteamientos de Dewey (1995), Argyris (1980), Schön (1998), Zeichner (1982), Barnett (1992), Perrenoud (2007), Domingo (2012), Anijovich (2009), Pedroza (2014) y Cerecero (2018), puede afirmarse que la práctica reflexiva se caracteriza por ser un proceso ético, consciente, intencional, metódico, deliberativo, social, que requiere tiempo y supone un distanciamiento de la actividad para pensar en esta, conocerla y reconocerla, así como permitir al profesional conocerse y reconocerse; compara y conecta teoría y práctica, exige una suspensión del juicio para buscar la objetividad; implica actuar y transformar, busca el desarrollo humano y profesional de quien la ejerce, e involucra aceptar la responsabilidad de las decisiones tomadas.

\section{MODELOS QUE APOYAN A LA PRÁCTICA REFLEXIVA}

Esto modelos son entendidos como aquellos esquemas teóricos, que pueden o no estar clasificados dentro de la práctica reflexiva, pero que apoyan su proceso en uno o más aspectos. Algunos se describen a continuación: 


\section{Modelo de Investigación-Acción en Espiral}

Este modelo fue propuesto por Kurt Lewin en 1946 (Mertler, 2009), aplicándolo principalmente en acciones de apoyo comunitario. Se inicia por un ciclo que involucra encontrar o definir los hechos, planear, actuar, evaluar y modificar el plan, según se considere necesario, extendiéndose al terminar un ciclo en otros ciclos más. Ha sido representado de la siguiente manera:

Figura 1. Modelo de investigación-acción en espiral de Lewin.

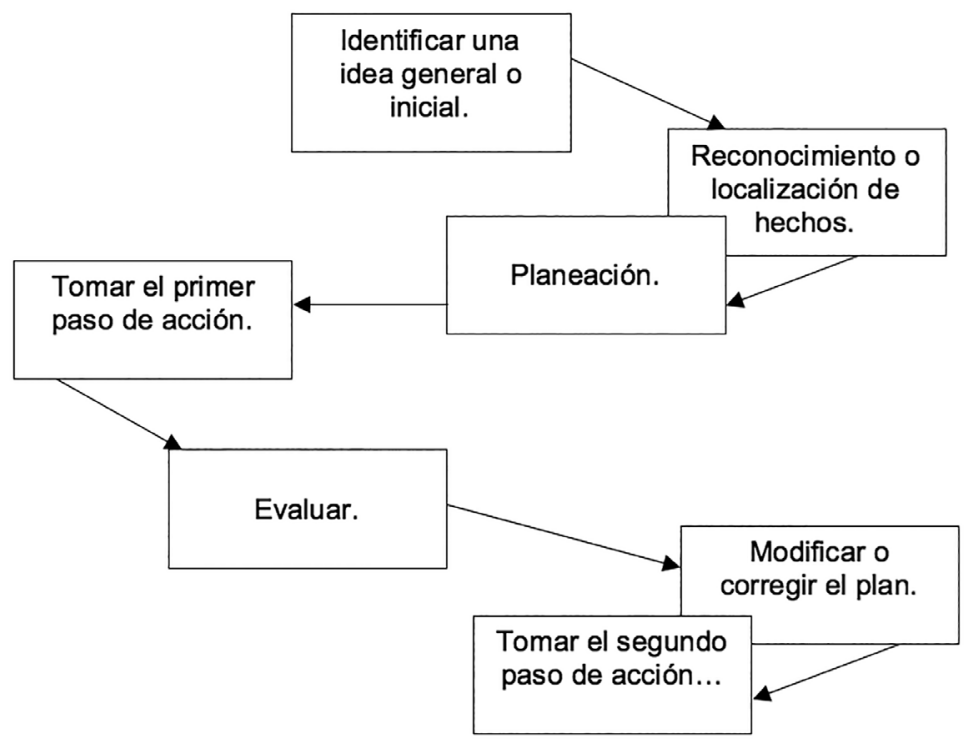

Fuente: Traducido de Mertler (2009, p. 14).

\section{Modelo de Investigación-Acción de Mertler}

Este modelo es cíclico y posee cuatro etapas: planear, actuar, desarrollar y reflexionar; cada una sigue determinados pasos: 
Planeación. 1) Se identifica y limita el tema, es decir, se decide lo que se va a estudiar, ya sea por un interés personal o por una necesidad presente. 2) Se reúne información, a partir de otras personas que pueden dar cierta retroalimentación porque conocen del tema o se encuentran en nuestro contexto. 3) Se revisa la literatura o cualquier fuente de información que proporcione datos confiables de manera que pueda contrastarse teoría y práctica. 4) Se desarrolla un plan de investigación, definiendo una metodología a seguir e iniciando con el establecimiento de una pregunta de investigación, una hipótesis o supuesto teórico, las variables y características a medir o analizar, y el seguimiento que se dará a la investigación a lo largo del proceso.

Acción. En esta etapa se especifican los datos a recolectar y las técnicas para recolectarlos, por ejemplo: observación, notas de campo, entrevistas, etcétera. Los pasos de esta etapa son: 1) Recolectar los datos. 2) Analizar los datos conforme se obtienen, de manera que surjan nuevos temas o categorías y el investigador pueda integrar o modificar su búsqueda; y un análisis más, al final del proceso, cuando ya se tienen todos los datos de manera que puedan interpretarse.

Desarrollo. Una vez analizados e interpretados los datos, se desarrolla el plan de acción para probar las nuevas ideas propuestas y trabajar en resolver el problema. Este plan de acción es puesto en práctica y debe ser monitoreado, evaluado y revisado continuamente para preservar la naturaleza cíclica de la investigación-acción y efectivamente resolver el problema, así como descubrir cualquier otra opción o problema.

Reflexión. Finalmente, se sugiere: 1) Compartir y comunicar los resultados dentro del contexto en que se llevó a cabo, presumiendo que los problemas que se presentan pueden presentarse no solo para quien los investigó, sino para cualquier otro de sus colegas; o incluso compartirse en comunidades externas que pueden beneficiarse porque comparten los ejercicios de la profesión. 2) Se reflexiona acerca del proceso, revisando lo hecho, su efectividad y la posibilidad de tomar decisiones para nuevas implementaciones en el proyecto. 
Figura 2. Modelo de investigación-acción de Mertler.

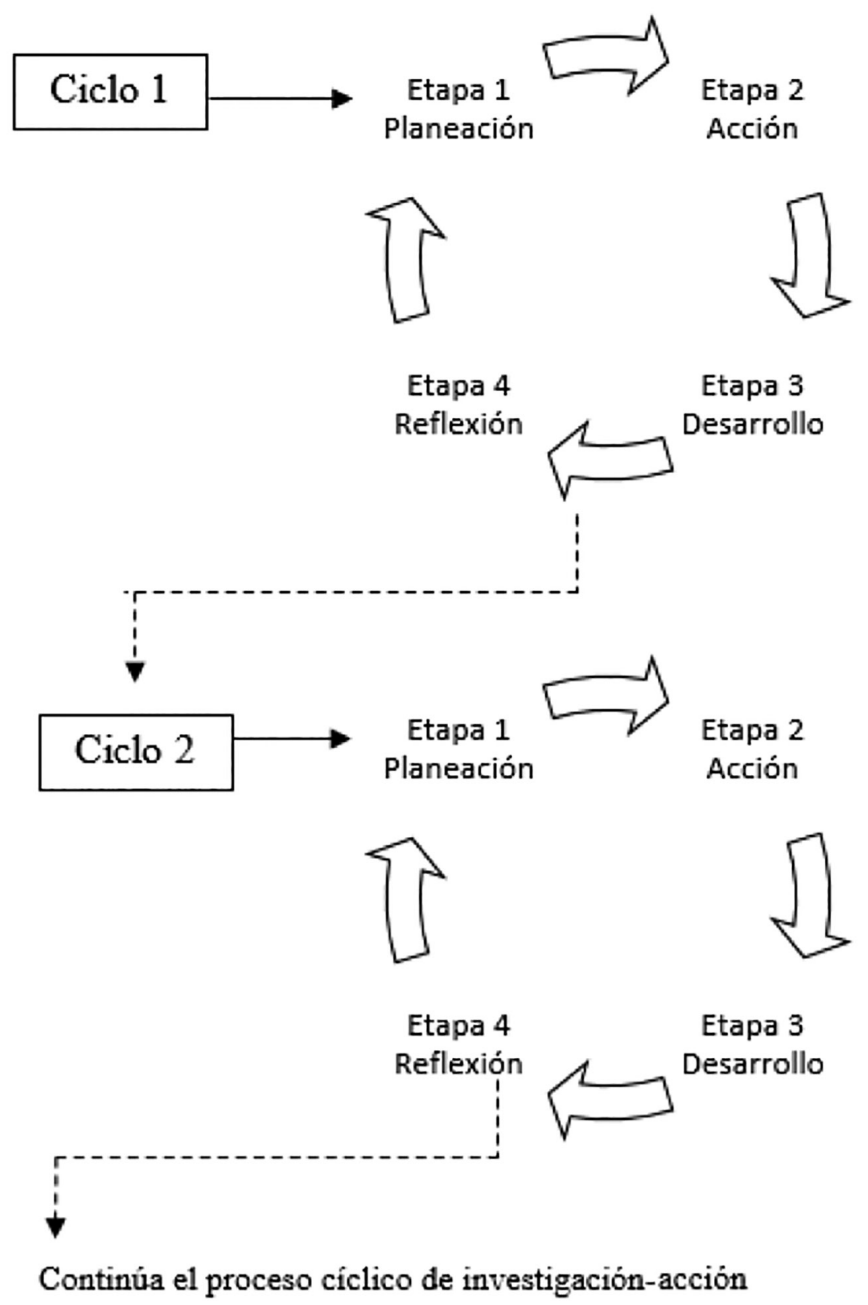

Fuente: Adaptado de Mertler (2009, p. 32). 


\section{3-4. Modelos de Aprendizaje de una Sola Vuelta y de Doble Vuelta}

Argyris y Schön proponen dos modelos: el modelo de aprendizaje de una vuelta (o single loop learning) y el modelo de aprendizaje de doble vuelta (o double loop learning). El primero se enfoca en la solución de un problema sin importar su origen: se busca una solución operativa, evitando el análisis o cambio de variables. El segundo, además de identificar el problema, cuestiona su origen y hace cambios en las variables para observar sus consecuencias y encontrar una solución más adecuada y con mayor permanencia.

Figura 3. Aprendizaje de una vuelta (single loop learning).

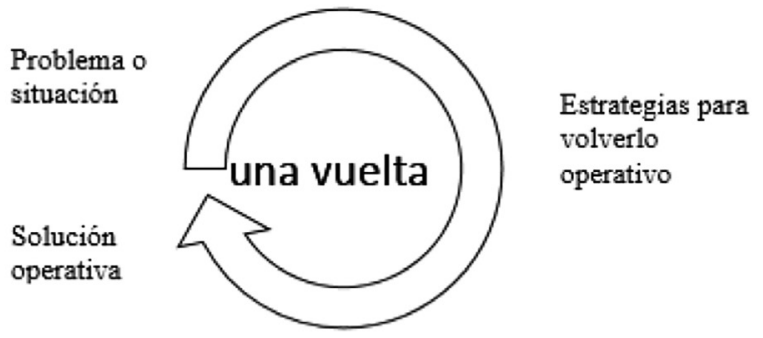

Fuente: Adaptado de Argyris y Schön (1978).

Figura 4. Aprendizaje de dos vueltas.

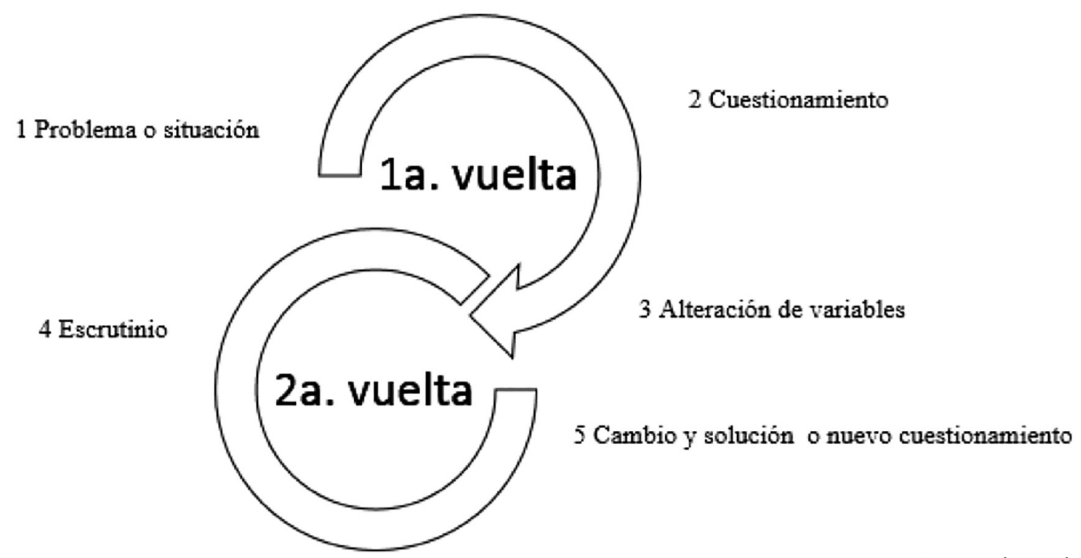

Fuente: Adaptado de Argyris y Schön (1978). 


\section{Modelo Reflexivo de Rolfe, Freshwater y Jasper}

Este modelo ofrece una manera simple de reflexión de los hechos. Se basa en tres preguntas fundamentales, las cuales pueden irse ampliando para obtener un mayor detalle del problema, estas preguntas son: ¿qué o cuál?, ¿así que?, y ¿ahora qué?

La primera pregunta corresponde a la descripción del evento y la autoconsciencia del mismo, por tanto esta podría ser completada de la siguiente manera: ¿Cuál es el problema? Pudiendo originar otras cómo: ¿Cuál es mi papel en esta situación? ¿Cuáles fueron las consecuencias? ¿Qué sentimientos se provocaron en mí y en los afectados? ¿Qué fue lo positivo y lo negativo de esta experiencia? ¿Qué podría mejorarse?

La segunda pregunta sirve para analizar la situación y evaluar las circunstancias expuestas en la primera. Ejemplos de preguntas en esta etapa son: ¿Así que esta situación que se ha presentado, qué me dice? ¿Así que en qué basé mis acciones? ¿Así que, qué hubiera pasado si hubiera actuado diferente? etcétera.

La tercera pregunta se centra en sintetizar la información para observar los cambios que pueden realizarse ante una situación similar. Pueden utilizarse preguntas como: ¿Ahora qué necesito hacer para arreglar la situación? ¿Ahora a quién podría solicitar su apoyo? ¿Ahora qué he aprendido de la experiencia? ¿Ahora qué debo evitar en un futuro?, entre otras.

Este modelo no toma en cuenta ninguna teoría, se basa únicamente en la reflexión de la experiencia; se sugiere escribirlo y no requiere de ningún acompañamiento ni de otras personas para llevarlo a cabo. 


\section{Figura 5. Modelo de reflexión de Rolfe, Freshwater y Jasper.}

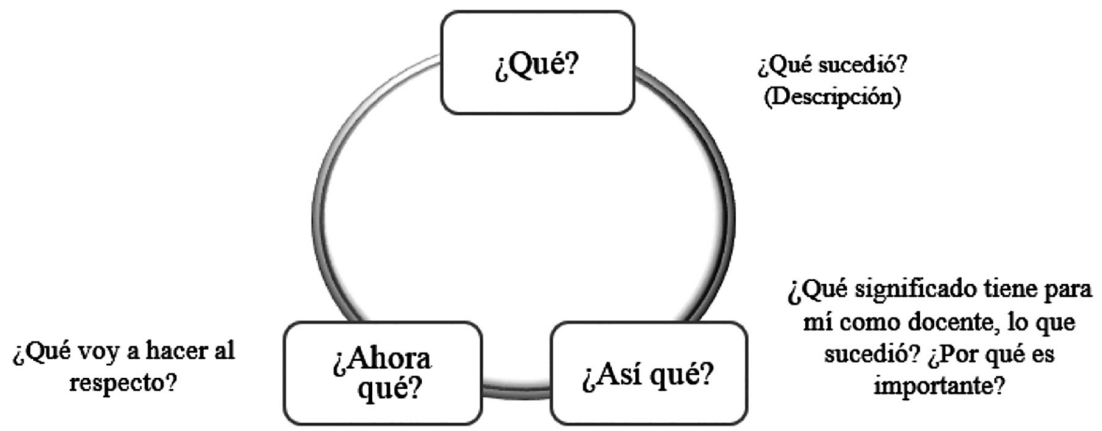

Fuente: Adaptado de Rolfe, Freshwater y Jasper (2010).

\section{Modelo del Ciclo de Aprendizaje Experiencial de Kolb}

En 1984, David A. Kolb (McLeod, 2013) presenta un modelo reflexivo cíclico basado en su teoría de aprendizaje experiencial que pretende ser consistente con la forma de aprendizaje natural a través de la experiencia. Este modelo considera cuatro etapas:

Experiencia concreta. Actuar o llevar a cabo una tarea asignada.

Observación reflexiva. Tomarse un tiempo para pensar en lo que se ha experimentado, formular preguntas y discutir lo sucedido con los demás que lo han experimentado.

Conceptualización abstracta. Es el proceso de entender lo sucedido e interpretar los eventos y relacionarlos con los conocimientos previos que provienen de la teoría estudiada, de otras personas expertas o involucradas, de observaciones previas o de cualquier otro conocimiento desarrollado en el pasado.

Experimentación activa. Plan de acción que incluye las predicciones de lo que se espera suceda y de las acciones que deberán realizarse como consecuencia. Finalmente, se pone en acción lo aprendido para ver su utilidad y que pueda ser recordado. 
Figura 6. Aprendizaje experiencial de Kolb.
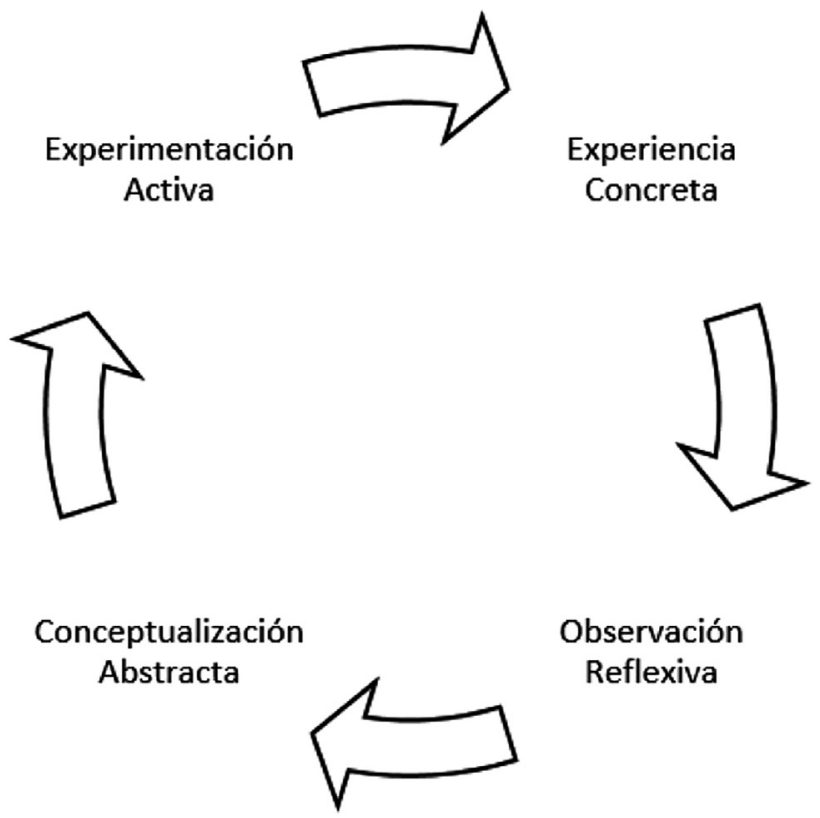

Observación

Reflexiva

Fuente: Adaptado de McLeod (2013).

\section{Modelo Reflexivo de Gibbs}

En 1988, Graham Gibbs propone un ciclo reflexivo en su obra Aprender haciendo. Este modelo parte de una experiencia o situación sobre la que se quiere reflexionar y consta de seis elementos:

Descripción. Relatar detallada y objetivamente el problema o suceso a reflexionar, preferentemente de forma escrita.

Sentimientos. Identificar los sentimientos experimentados durante y después del suceso, observando si se alteraron y describiéndolos con la mayor exactitud posible. 
Evaluación. Valorar cómo se reaccionó y actuó en la situación descrita. Se identifican los puntos a favor y en contra, los resultados del evento citado, cómo se resolvió, a qué conclusión se llegó, así como su efectividad.

Análisis. Examinar cada uno de los aspectos cruciales, comparándolos con la teoría relacionada para entender y explicar lo sucedido.

Conclusión. Con base en los resultados de análisis, se generan conclusiones sobre lo acontecido y se consideran algunas alternativas de solución que pudieran haberse tomado en cuenta para un desenlace más favorable.

Plan de acción. Se toman decisiones sobre lo que se hará en caso de presentarse un problema similar al experimentado, de manera que los resultados sean positivos y eviten el problema presentado.

Figura 7. Ciclo reflexivo de Gibbs.

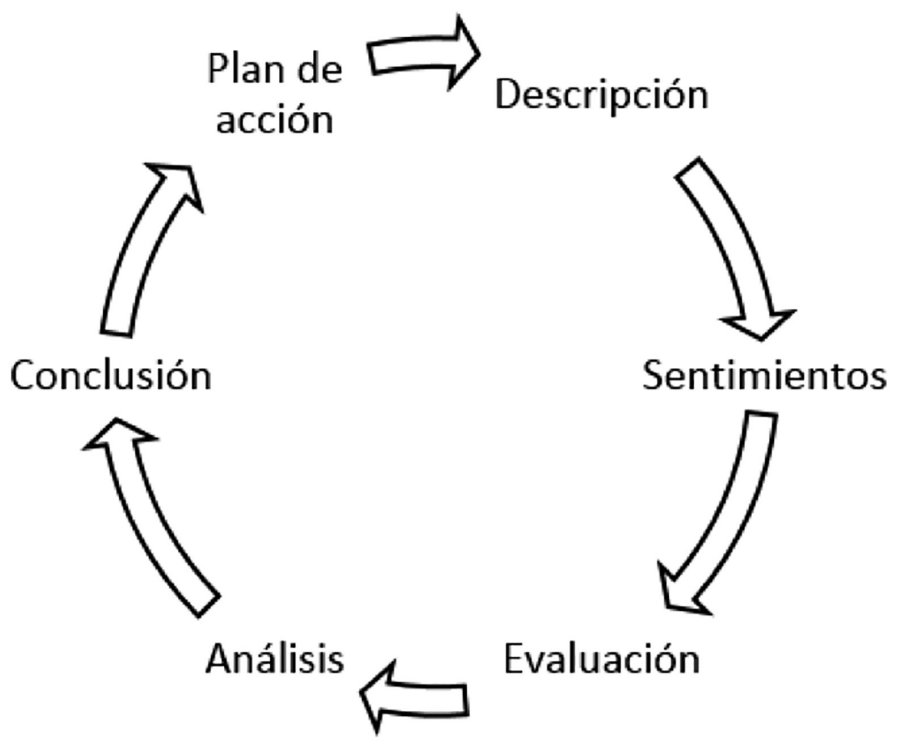

Fuente: Adaptado de University of Bradford (2003). 


\section{Modelo de la Teoría del Aprendizaje Expansivo}

De acuerdo con Engeström (2010), en el aprendizaje expansivo se aprende algo nuevo a través de su construcción, conceptualización e implementación en la práctica. Esta teoría aplica no solo para el aprendizaje, sino para la mejora docente o profesional. Como puede verse en la imagen, inicia con el cuestionamiento que permite conocer las necesidades para, posteriormente, analizar los datos obtenidos considerando los antecedentes del problema y la situación presente; a partir de los descubrimientos del análisis se buscan propuestas de solución, creándose un posible modelo que es, posteriormente, llevado a la práctica para probarlo, evaluarlo y hacer los ajustes correspondientes; finalmente se reflexiona sobre el proceso y buscando su consolidación al incorporarse de manera continua en la práctica. Una vez terminado el ciclo se puede reiniciar con nuevas preguntas de mejora para el mismo suceso o para otro que se quiera mejorar.

\section{Figura 8. Secuencia de acciones de aprendizaje en un ciclo de aprendizaje expansivo según Engëstrom.}

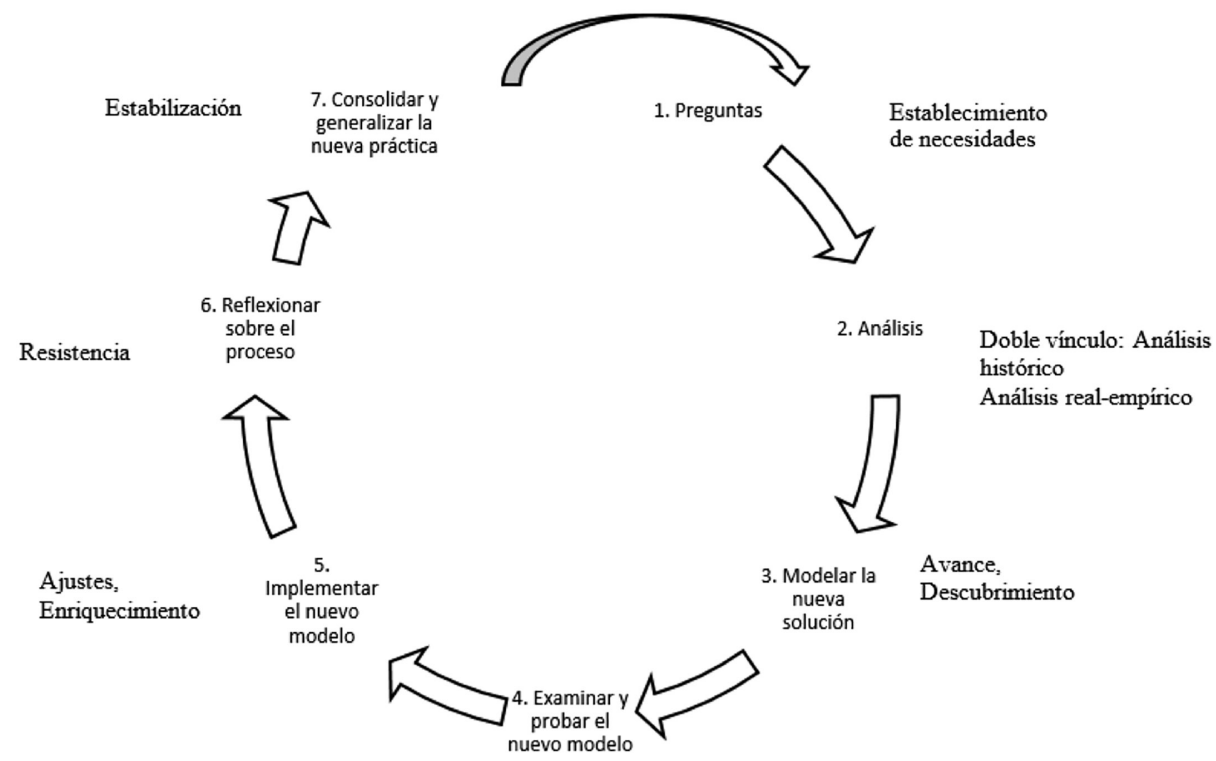

Fuente: Engëstrom (2010, p. 80). Traducción propia. 


\section{Modelo R5}

Domingo y Gómez (2014) proponen un modelo de práctica reflexiva que consiste en cuatro o cinco fases para mejorar la práctica del docente, a partir de la reflexión individual (Modelo R4) o grupal (Modelo R5), respectivamente.

R1. Elección de una experiencia que se desea analizar, sin importar si su resultado fue de éxito o fracaso.

R2. Describe lo sucedido a detalle de forma escrita, de manera que se reconstruya la experiencia.

R3. Reflexión individual autorregulada donde se parte del conocimiento de lo sucedido y del conocimiento previo o formativo del profesional; se analiza la reflexión durante la acción y se reflexiona sobre esa reflexión durante la acción, con el propósito de mejora en caso de que se volviera a presentar la intervención descrita.

R4. La cuarta fase se considera opcional: se procede a una reflexión compartida o grupal, con otras personas involucradas o con la teoría existente, de manera que se pueda comparar y contrastar la información obtenida, a modo de retroalimentación.

R5. La última fase consiste en planificar lo que se hará a fin de mejorar la práctica, tomando en cuenta lo aprendido de la situación sobre la que se reflexionó, lo que se desea modificar o conservar de la misma, los conocimientos teóricos relacionados y los vacíos intelectuales detectados. 
Figura 9. Modelo R5.

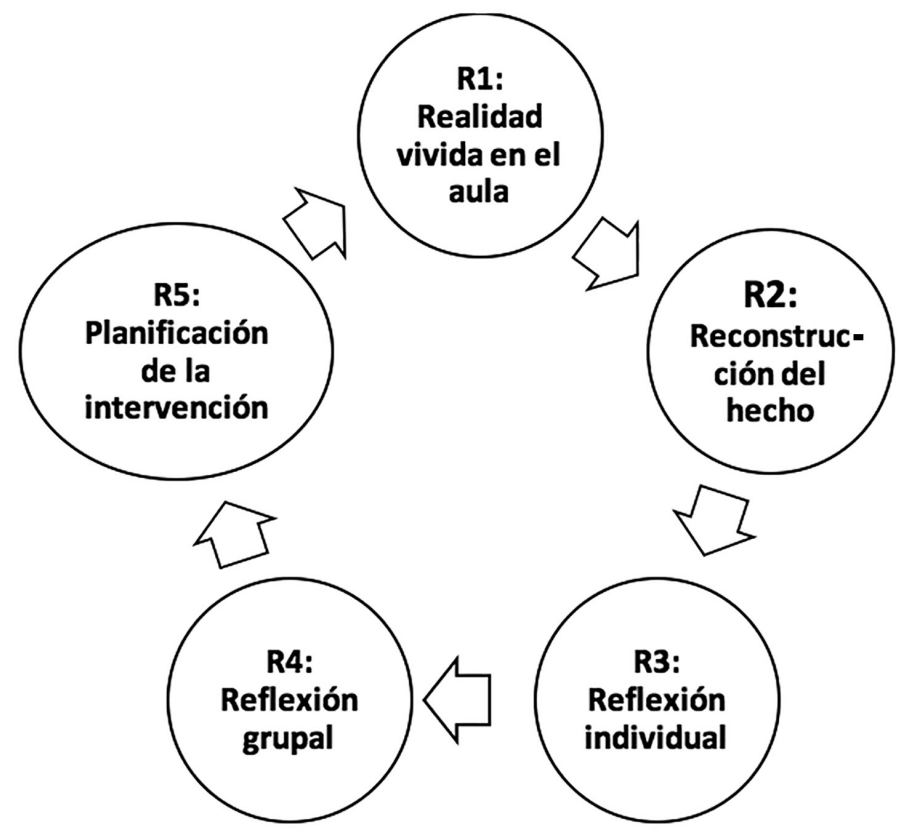

Fuente: Domingo y Gómez (2014).

\section{Modelo de Práctica Reflexiva Mediada}

En este modelo cíclico de reflexión sobre la praxis del profesional y su autoconocimiento, Cerecero (2018) propone incorporar tres elementos base: 1) La mediación de un tutor como acompañamiento del profesional que lleva a cabo el proceso reflexivo, así como el apoyo de otros agentes, y del contraste entre la práctica y la teoría. 2) La búsqueda del autoconocimiento del profesional, el conocimiento de su práctica y de su contexto. 3) La transformación real y perceptual del profesional en su acción. De este modo, el modelo consta de tres fases cada una con tres ciclos:

Fase 1. Conocimiento. El cual incluye: 1) El autoconocimiento del profesional, de su práctica y de su contexto. 2) El conocimiento de sí mismo, su práctica y su contexto, a través de los demás; y 3) La interpretación al contrastar la información obtenida en los dos ciclos anteriores. 
Fase 2. Significación. Una vez obtenida la información de la primera fase: 1) Se identifica y plantea un problema, describiéndolo detalladamente. 2) Se busca información para fundamentar y contrastar la experiencia con la teoría (análisis de los datos). 3) Se elabora un plan de acción que sintetiza los aprendizajes obtenidos en los ciclos anteriores.

Fase 3. Resignificación. Con el fin de resignificar la práctica se requiere de: 1) Llevar a la práctica el plan de acción. 2) Evaluar los resultados de la intervención, reflexionando sobre ellos y elaborando un aporte teórico sobre el proceso y las conclusiones a las que se haya llegado. 3) Evaluar si se alcanzó una transformación de la práctica, no solo en la acción, sino también a nivel mental en la forma de percibir los hechos.

Figura 10. Modelo de práctica reflexiva mediado.

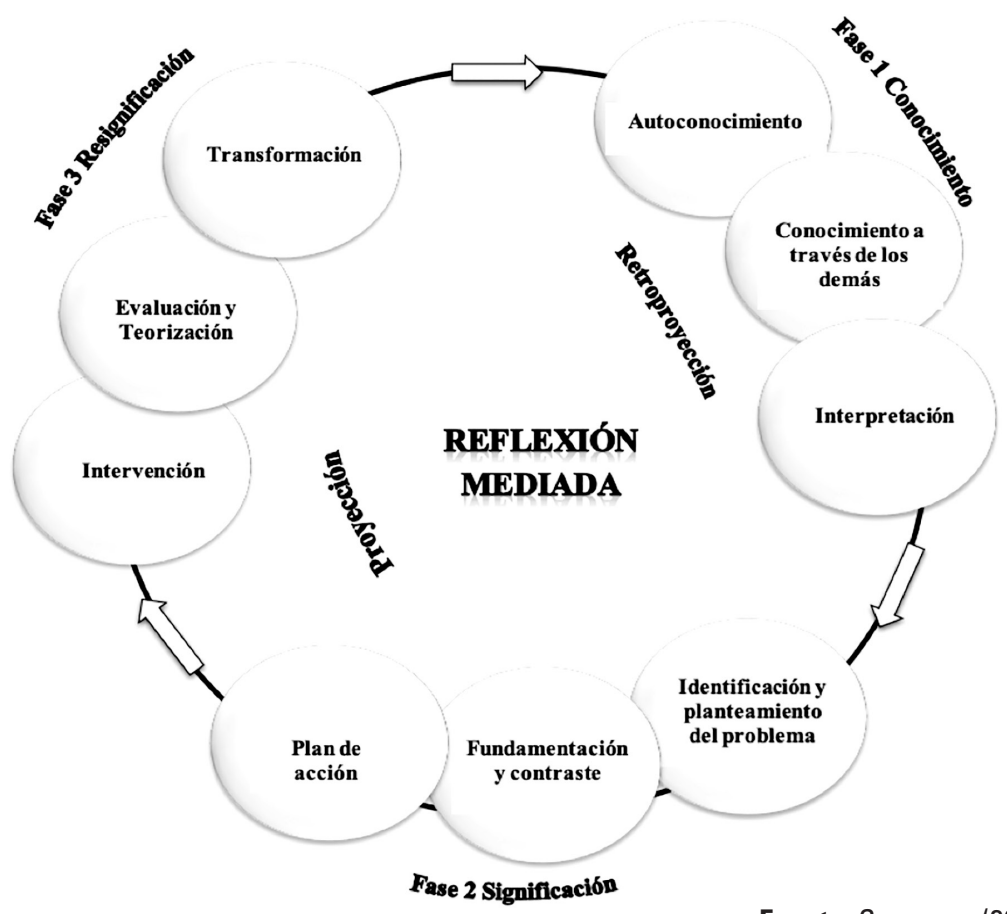

Fuente: Cerecero (2018). 


\section{CONCLUSIONES}

Mucho se ha hablado de la necesidad de ser críticos y reflexivos en las últimas décadas, siendo estas habilidades consideradas como uno de los desafíos y soluciones, no solo para los procesos de enseñanza-aprendizaje, sino también en la proyección donde cada uno de los distintos profesionales se desempeñan, sin importar el área en que estén inmersos.

Es a través de la reflexión que pueden mejorarse las acciones y los resultados de las experiencias en el ámbito laboral, social y personal. Sin embargo, una reflexión eventual no tendrá los mismos resultados que una práctica reflexiva, puesto que esta última implica un proceso introspectivo sistemático, intencional que busca comprender cada una de las situaciones trascendentales que se van presentando o creando para analizar sus efectos, comprenderlos, corregirlos, perfeccionarlos o transformarlos.

Una vez estudiados algunos de los modelos que apoyan a la práctica reflexiva, se observa que la mayoría son cíclicos y parten de la detección de un problema o situación a resolver; algunos se concentran únicamente en el análisis y la comprensión de la experiencia, como son: el método de investigación-acción en espiral; los modelos de aprendizaje de una o doble vuelta; el modelo reflexivo de Rolfe, Freshwater y Jasper y el R4. Mientras que otros modelos buscan un fundamento teórico para contrastar, como el método R5; todos implican una decisión de acción futura, en general envuelven el diseño de un plan de acción, su puesta en escena y su evaluación.

Sin embargo, algunos modelos son operativos y superficiales, por ejemplo: el aprendizaje de una vuelta de Argyris y Schön, o bien el modelo reflexivo de Rolfe, Freshwater y Jasper; mientras que otros buscan mayor profundidad y soporte, como el modelo de investigación acción de Mertler, el R5 y el de la Práctica Reflexiva Mediada.

Por lo anterior, se concluye que una práctica reflexiva va más allá del uso de un modelo. Supone un estilo de vida laboral que involucra el autocuestionamiento, la autoconciencia, la observación de la práctica y del contexto, el análisis de las experiencias y de los hechos, su 
comprensión e interpretación, estar dispuestos al cambio, a reaprender y a aceptar la responsabilidad de las decisiones y acciones tomadas; y sobre todo, la búsqueda continua de una mejora de uno mismo y del medio en que se desenvuelve, no solo para la transformación personal sino para el bien común.

\section{REFERENCIAS}

Anijovich, R. (2009). Transitar la formación pedagógica: Dispositivos y estrategias. Buenos Aires: Paidós.

Argyris, C. (1980). Inner contradictions of rigorous research. New York: Academic Press.

Argyris, C., \& Schön, D. A. (1978). Organizational learning: A theory of action perspective. Reading, Massachusetts: Addison-Wesley.

Atkinson, S. (Ed.). (2011). El libro de la Filosofía. Madrid, España: Ediciones Akal/Darling Linderley Limited.

Barnett, R. (1992). Improving Higher Education. Buckinham: SRHE/ Open University Press.

Cerecero, I. (2018). Práctica Reflexiva Mediada: del autoconocimiento a la resignificación conjunta de la práctica docente. Mauritius: Editorial Académica Española.

Cerecero, I. (2018, junio 1). «Propuesta de un nuevo modelo: Práctica Reflexiva Mediada». INNOEDUCA, International Journal of Technology and Educational Innovation, 4(1), 44-53.

Dewey, J. (1993). Cómo pensamos: Nueva exposición de la relación entre pensamiento y proceso educativo (A. C. Barcelona), Trans.) Barcelona: Paidós.

Dewey, J. (1995). Democracia y educacion: Una introducción a la filosofía de la educación (2a ed.). Madrid: Ediciones Morata. 
Domingo Roget, Á., y Gómez Serés, M. V. (2014). La Práctica Reflexiva: Bases, modelos e instrumentos. Madrid: Narcea.

Domingo, A. (2012). ¿Qué es la Práctica Reflexiva?. Recuperado de Práctica Reflexiva: https://practicareflexiva.pro/que-es-la-practica-reflexiva/

Ediciones, T. d. (Ed.). (2015). Recuperado de Diccionario de psicología Científica y filosófica: http:// www.e-torredebabel.com/Psicologia/ Vocabulario/Duda-Metodica.htm

Engëstrom, Y. (2010). Activity theory and learning at work (U. o. Helsinky, Ed.) Recuperado de Center for Activity Theory and Developmental Work Research: http:// www.helsinki.fi/cradle/documents / Engestrom\%20Publ/Chapter\%20for\%20Malloch\%20book.pdf

García, A. D. (1999). «Introducción a la fenomenología de Edmund Husserl». Recuperado de Revista de ciencias humanas: http://www. utp.edu.co/ chumanas/revistas/revistas/rev22/garcia.htm

Lantolf, J. P. (2007). Praxis and classroom L2 development. Recuperado de Researchgate.net: https://www.researchgate.net/profile/ James Lantolf/publication/28249595 Praxis and Classroom L2 Development/links/0c960534d4ff86f10f000000.pdf?origin=publication detail\&ev=pub int prw xdl\&msrp=7k3TF1ruX9WNfRvcmkFQMvZRWIeCvLv62jheZVNvXhS8cGV5loLwY8Uvh-kGlI

León, E. (2012). «El giro hermenéutico de la fenomenológica en Martín Heidegger» (C. d. (CISPO), Ed.) Recuperado de Polis: file: / / C: / Users / ASUS\% 20VIVOBOOK/Downloads/polis-2690-22-el-giro-hermeneutico-de-la-fenomenologica-en-martin-heidegger.pdf

Lewin, K. (1946). «Action research and minority problems». Journal of Social Issues, 2, 34-46.

Lisón, J. F. (1991). Reflexión (Filosofía). (E. Rialp, Ed.) Recuperado de Mercabá: http:/ / www.mercaba.org/Rialp/R/ reflexion filosofia.htm

McLeod, S. (2013). «Kolb - Learning Styles». Recuperado de Simply Psychology : http:// cei.ust.hk/files/public/simplypsychology kolb learning styles.pdf 
Mertler, C. A. (2009). Action Research: Teachers as Researchers in the Classroom (2nd. ed.). USA: SAGE Publications.

Moon, J. (1999). Reflection in learningand professional development: Theory and practice. London: Kogan.

Muñoz, J., y Velarde, J. (2000). Compendio de Epistemología. Madrid: Trotta.

Pedroza Flores, R., Villalobos Monroy, G., y Nava Gómez, N. (2014). Un método para la práctica educativa, afectiva y creativa. México: Universidad Autónoma del Estado de México / MAPorrúa.

Pedroza, R. (2014). La Investigación-Acción en la Práctica Educativa Reflexiva (2a ed.). México: Colegio de Investigadores en Ciencias de la Educación.

Perrenoud, P. (2007). Desarrollar la práctica reflexiva en el oficio de enseñar: Profesionalización y razón pedagógica (4a. ed.). Barcelona: Graó.

Rolfe, G., Freshwater, D., \& Jasper, M. (2001). Critical reflection in nursing and the helping professions: a user's guide. Basingstoke: Palgrave Mcmillan.

Schön, D. A. (1992). La formación de profesionales reflexivos: Hacia un nuevo diseño de la enseñanza y el aprendizaje en las profesiones. Barcelona: Ediciones Paidós y Ministerio de Educación y Ciencia.

Schön, D. A. (1998). El profesional reflexivo: Cómo piensan los profesionales cuando actúan. Barcelona: Paidos.

Torres, H. S., y Miranda, A. (2013). «¿Es necesaria la categoría de reflexión en la psicología?». Revista electrónica de psicología Iztacala, 16(1), 34-45. Recuperado de http:// www.iztacala.unam.mx/carreras/psicologia/psiclin/vol16num1/Vol16No1Art3.pdf

University of Bradford (2003). Reflection Models and frameworks. Recuperado de University of Bradford: https://www.bradford.ac.uk/ wimba-files/skill-space/Reflective Writing HTML/page 04.htm

Zeichner, K. M. (1982, mayo 8). Factors related to reading performance. Recuperado de Práctica Reflexiva: http://www.practicareflexiva. pro/wp-content/ uploads / Org-El-maestro-como-profesional-reflexivo-de-Kenneth-M.-Zeichner..pdf 